\title{
Verbreitung zytologischer Verfahren in der bronchoskopischen Diagnostik peripherer pulmonaler Herde
}

\section{Frequency of Cytological Procedures in Diagnostic Bronchoscopy of Peripheral Pulmonary Modules and Masses}

Autoren

Institute
K.-J. Franke, G. Nilius, K.-H. Rühle

Klinik für Pneumologie, Allergologie und Beatmungsmedizin, Klinik Ambrock, Hagen, Universität Witten/Herdecke (Professor Dr. med. Karl-Heinz Rühle) eingereicht 12.6. 2006

akzeptiert 28.6.2006

\section{Bibliografie}

Dol $10.1055 / \mathrm{s}-2006-944263$

Pneumologie 2006; 60; 663-666

(c) Georg Thieme Verlag KG

Stuttgart · New York

ISSN 0934-8387

Korrespondenzadresse Dr. med. Karl-Josef Franke Klinik für Pneumologie Ambrock

Ambrocker Weg 60

58091 Hagen

franke@klinik-ambrock.de

\section{Zusammenfassung}

Hintergrund: Durch Hinzunahme von Methoden zur Gewinnung zytologischen Materials zur transbronchialen Zangenbiopsie wird die Sensitivität in der Diagnostik des peripheren Bronchialkarzinoms erhöht. Eine möglicherweise in Vergessenheit geratene effektive zytologische Technik stellt die transbronchiale Katheteraspiration dar. Zur Verbreitung zytologischer Verfahren sowie zum Stellenwert der transbronchialen Katheteraspiration im Vergleich zu transbronchialer Nadelaspiration und Bürstenabstrich führten wir eine Umfrage durch.

Methode: An sämtliche 99 Pneumologische Kliniken in Deutschland mit zweijähriger Weiterbildungsermächtigung im Fach Pneumologie wurden Fragebogen gesandt.

Ergebnisse: Wir erhielten einen Rücklauf von 70 Fragebogen (69\%). 79\% der Kliniken kombinieren die Zangenbiopsie mit zytologischen Methoden. Der Bürstenabstrich stellt mit $51 \%$ die am meisten verbreitete zytologische Technik dar gegenüber nur je $28 \%$ für die transbronchiale Katheteraspiration und die transbronchiale Nadelaspiration. Bei regelmäßiger Bronchiallavage durch $47 \%$ der Untersucher führt nur gut ein Viertel (27\%) regelhaft eine Imprintzytologie durch. Schlussfolgerungen: Der überwiegende Anteil der befragten Kliniken setzt regelmäßig kombiniert zytologische Verfahren ein, was aufgrund ihrer hohen Spezifität und einer Erhöhung der Trefferquote im Vergleich zur transbronchialen Zangenbiopsie alleine sinnvoll ist. Transbronchiale Katheter- und Nadelaspiration finden relativ selten Verwendung, obwohl es sich hier um die einzigen zytologischen Verfahren mit einer signifikant höheren Trefferquote als der der Zangenbiopsie handelt.

\section{Abstract}

Background: The combination of forceps biopsy and cytological methods improves the yield in diagnostic bronchoscopy of peripheral lung cancer. Transbronchial catheter aspiration seems to be an underutilized cytological technique, which is valuable and minimally invasive. We inquired for the frequency of cytological procedures and the importance of transbronchial catheter aspiration in comparison to transbronchial needle aspiration and bronchial brushing.

Methods: We sent questionnaires to 99 clinically practising and educating pulmonologists.

Results: The response amounted to $69 \%$. Nearly $79 \%$ of the pulmonologists usually combine forceps biopsy with cytological procedures. Instead of $51 \%$ bronchial brushing, transbonchial catheter aspiration as well as needle aspiration is the most applied cytological technique by only $28 \%$ of the interrogated physicians. $47 \%$ of the investigators practise bronchial washing and 27\% practise core roll preparations as a rule.

Conclusions: Most of the specialized clinics in respiratory medicine in Germany add cytological methods to transbronchial forceps biopsy. This practice is pregnant with meaning because of a higher diagnostic yield while accuracy is likewise high. Unlike bronchial brushing transbronchial catheter aspiration and needle aspiration are underutilized for different reasons in spite of the fact, that these are the only procedures with a significant higher sensitivity in comparison to forceps biopsy. 


\section{Einleitung}

Um zu einer Gewebebeurteilung peripherer Lungenherde zu gelangen, wird häufig die flexible Bronchoskopie angewandt. Sie stellt ein effektives und sicheres Untersuchungsverfahren dar, das nicht selten Patienten vor operativen Prozeduren bewahrt. Die Trefferquote nimmt allerdings bei Herden $<3 \mathrm{~cm}$ und im äußeren Thoraxdrittel gelegenen Läsionen ab [1]. Techniken zur Gewinnung sowohl histologisch als auch zytologisch zu untersuchenden Materials werden eingesetzt. Ein verbreitetes histologisches Verfahren stellt die transbronchiale Zangenbiopsie (TBB) dar. Eine Erhöhung der Trefferquote durch Hinzunahme von Methoden zur Gewinnung zytologischen Materials ist durch mehrere Untersuchungen belegt ( Tab. 1). Als Methoden zur Gewinnung zytologischen Materials sind die transbronchiale Nadelaspiration (TBNA), der Bürstenabstrich (BB), die Bronchiallavage (BW) und die transbronchiale Katheteraspiration (TBKA) zu nennen. In Bezug auf die Verlässlichkeit zytologischer Verfahren besteht in der Diagnostik des Bronchialkarzinoms eine generelle Akzeptanz [2,3]. Die Technik der transbronchialen Katheteraspiration wurde 1961 von Friedel eingeführt [4]. Trotz einer hohen diagnostischen Sensitivität wurde zu diesem Verfahren 25 Jahre lang nicht publiziert [5].

Unter folgender Fragestellung führten wir eine Umfrage unter erfahrenen Pneumologen durch:

1. Wie ist die Verbreitung zytologischer Verfahren in der bronchoskopischen Diagnostik peripherer Lungenherde in Deutschland?

2. Welchen Stellenwert hat die transbronchiale Katheteraspiration im Vergleich zur transbronchialen Nadelaspiration und zum Bürstenabstrich?

\section{Methodik}

Wir werteten den Rücklauf von Fragebogen aus, die an alle 99 Pneumologischen Kliniken in Deutschland mit zweijähriger Weiterbildungsermächtigung im Fach Pneumologie gesandt worden waren. Die Antworten auf folgende Multiple-ChoiceFragen wurden notiert:

1. Kombinieren Sie in der bronchoskopischen Diagnostik peripherer Rundherde regelmäßig zytologische und histologische Verfahren?
a) Ja
b) Nein

2. Welches der folgenden zytologischen Verfahren wenden Sie am häufigsten an?
a) Bürstenabstrich
b) Katheteraspiration
c) Trans-

bronchiale Nadelaspiration

3. Führen Sie regelmäßig eine Bronchiallavage zur zytologischen Diagnostik peripherer Lungenherde durch?
a) Ja
b) Nein

4. Wenden Sie regelmäßig das Verfahren der Imprintzytologie an?
a) Ja
b) Nein

\section{Ergebnisse}

70 Fragebogen wurden ausgefüllt zurückgesandt (69\%). Die Antworten zu Frage 2 sind in Tab. 2 wiedergegeben. Auf 8 Bögen war diese Frage nicht beantwortet. 12 Befragte kreuzten 2 und 1 Befragter alle 3 Möglichkeiten an. Die Summe der Prozentzahlen ergibt deshalb nicht 100 .

Die Fragen 1, 3 und 4 waren durchgehend mit Ja oder Nein beantwortet. Die Ergebnisse sind in Tab. 3 wiedergegeben.

\section{Diskussion}

Bei häufiger Kombination von Zangenbiopsie und zytologischen Methoden (78,6\%) an pneumologischen Fachkliniken in Deutschland ist der Bürstenabstrich mit $51 \%$ die am meisten verbreitete zytologische Technik zur Diagnostik peripherer pulmonaler Läsionen. Die transbronchiale Katheteraspiration erfolgt gleichermaßen selten wie die transbronchiale Nadelaspiration (in je $28 \%$ der Kliniken häufigste zytologische Methode). Mehrfachantworten zu Frage 2 wurden berücksichtigt, um den Stellenwert der Verfahren genauer zu erfassen. Eine Bronchiallavage wird von etwa der Hälfte der Untersucher regelhaft durchgeführt; das Verfahren der Imprintzytologie, bei dem das Abtupfpräparat der Zangenbiopsie zusätzlich zytologisch untersucht wird, ist mit $27 \%$ bei genannter Indikation in Deutschland wenig repräsentiert ( Tab. 2 u. 3).

\section{Kombination}

Die Kombination von transbronchialer Zangenbiopsie und zytologischen Methoden ist sinnvoll, da die diagnostische Sensitivität in bezug auf das periphere Bronchialkarzinom erhöht wird ( Tab. 1). Gleichzeitig bestätigt eine Reihe von Arbeiten die hohe Spezifität zytologischer Verfahren beim peripheren Lungenkarzinom; die Rate falsch positiver Ergebnisse wird mit Werten zwischen $0 \%$ und maximal $1,7 \%$ angegeben [1,6-12].

Tab. 1 Diagnostische Sensitivität der Fiberbronchoskopie bei peripheren malignen pulmonalen Läsionen in verschiedenen Studien

\begin{tabular}{|c|c|c|c|c|c|c|}
\hline & $\begin{array}{l}\text { transbronchiale } \\
\text { Biopsie }\end{array}$ & $\begin{array}{l}\text { transbronchiale } \\
\text { Nadelaspiration }\end{array}$ & $\begin{array}{l}\text { Bürsten- } \\
\text { abstrich }\end{array}$ & $\begin{array}{l}\text { Katheter- } \\
\text { aspiration }\end{array}$ & $\begin{array}{l}\text { Bronchial- } \\
\text { lavage }\end{array}$ & $\begin{array}{l}\text { Kombina- } \\
\text { tion }\end{array}$ \\
\hline Mak u. Mitarb. 1990 [11] & $36,5 \% n=63$ & nicht erfolgt & $28,6 \% n=63$ & nicht erfolgt & $38,1 \% n=63$ & $55,6 \%$ \\
\hline Gasparini u. Mitarb. 1995 [8] & $53,9 \% n=404$ & $69,3 \% n=349$ & nicht erfolgt & nicht erfolgt & nicht erfolgt & $75,4 \%$ \\
\hline Katis u. Mitarb. 1995 [16] & $38 \% n=37$ & $62 \% n=37$ & $27 \% n=37$ & nicht erfolgt & $24 \% n=37$ & $70 \%$ \\
\hline Chechani 1996 [14] & $55 \% n=40$ & $50 \% n=37$ & $52 \% n=48$ & nicht erfolgt & $34 \% n=51$ & $77 \%$ \\
\hline Bilaceroglu u. Mitarb. 1998 [13] & $49 \% n=84$ & $57 \% n=84$ & $26 \% n=84$ & nicht erfolgt & $4 \% n=84$ & $68 \%$ \\
\hline Reichenberger u. Mitarb. 1999 [17] & $32 \% n=28$ & $46 \% n=114$ & $38 \% n=58$ & nicht erfolgt & $26 \% n=126$ & $\begin{array}{l}\text { nicht } \\
\text { angegeben }\end{array}$ \\
\hline Franke u. Mitarb. 2006 [5] & $50 \% n=26$ & nicht erfolgt & nicht erfolgt & $77 \% n=26$ & nicht erfolgt & $88 \%$ \\
\hline Baaklini u. Mitarb. 2000 [1] & $57 \% n=151$ & nicht erfolgt & $48 \% n=151$ & nicht erfolgt & $45 \% n=151$ & $64 \%$ \\
\hline
\end{tabular}


Tab.2 Umfrage an 99 pneumologischen Zentren in Deutschland mit einem Rücklauf von 70 Antworten. Häufigst angewandtes zytologisches Verfahren in der Diagnostik peripherer pulmonaler Herde (Mehrfachantwort möglich)

\begin{tabular}{|lll|} 
& $\begin{array}{l}\text { häufigstes zytologisches } \\
\text { Verfahren }\end{array}$ \\
& Anzahl & Prozent \\
\hline Bürstenabstrich & 36 & $51 \%$ \\
\hline transbronchiale Katheteraspiration & 20 & $28 \%$ \\
\hline transbronchiale Nadelaspiration & 20 & $28 \%$ \\
\hline
\end{tabular}

Tab. 3 Häufigkeiten der regelmäßigen Kombination von zytologischen und histologischen Verfahren, der regelmäßigen Anwendung einer Bronchiallavage sowie der Imprintzytologie zur bronchoskopischen Diagnostik des endoskopisch nicht sichtbaren Bronchialkarzinoms

\begin{tabular}{|lllll|} 
& ja & \multicolumn{2}{c|}{ nein } \\
& Anzahl & Prozent & Anzahl & Prozent \\
\hline $\begin{array}{l}\text { regelmäßige Kom- } \\
\text { bination Zytologie/ } \\
\text { Histologie }\end{array}$ & 55 & $78,6 \%$ & 15 & $21,4 \%$ \\
\hline $\begin{array}{l}\text { regelmäßige } \\
\text { Bronchiallavage }\end{array}$ & 33 & $47,0 \%$ & 37 & $52,9 \%$ \\
\hline $\begin{array}{l}\text { regelmäßige } \\
\text { Imprintzytologie }\end{array}$ & 19 & $27,0 \%$ & 51 & $72,9 \%$ \\
\hline
\end{tabular}

\section{Bürstenabstriche}

Zangenbiopsien, Bürstenabstriche und Nadelaspirationen geben morphologische Veränderungen unterschiedlicher anatomischer Areale wieder. So werden mit Bürstenabstrichen eher oberflächlich gelegene Veränderungen der Bronchialschleimhaut erfasst. Die Trefferquote beim peripheren pulmonalen Herd liegt für Bürstenabstriche zwischen 26 und $45 \%$ und nur selten signifikant über der der Bronchiallavage [1,11,13-16]. Gegenüber transbronchialer Zangenbiopsie und transbronchialer Nadelaspiration scheint der Bürstenabstrich das größere Risiko einer schweren Blutung zu beinhalten [14].

Eine Modifikation der Technik des Bürstenabstriches in der Diagnostik des peripheren pulmonalen Tumors stellen multiple Bürstenabstriche, kombiniert mit Sofortzytologie, ohne Führung mittels Röntgendurchleuchtung dar [9]. Die Spitze des Bronchoskopes wird so weit wie möglich in die nach vorherigem Studium der Röntgenbilder wahrscheinlichen Subsegmentostien vorgeschoben und sodann eine Nylonbürste so weit wie möglich im Bronchialsystem platziert. Die Trefferquote wurde mit $86 \%$ als deutlich über der in anderen Untersuchungen liegend bestimmt. Neben der Führung durch die Sofortzytologie kann als Erklärung gelten, dass die Bürste im Gegensatz zu Zange und Feinnadel Zellen aus einem relativ großen Bereich zu sammeln vermag. Allerdings wurde vergleichend in dieser Untersuchung keine andere Technik, insbesondere die transbronchiale Zangenbiopsie nicht, eingesetzt.

\section{Transbronchiale Katheteraspiration}

Über den in den Rundherd vorgeschobenen röntgendichten Katheter wird mechanisch Zellmaterial mobilisiert und aspiriert. Der Katheter kann mehrfach verwendet werden und ist dadurch preisgünstig. Das stumpfe distale Ende macht eine geringe Kom- plikationsrate und eine niedrige Gefahr einer Beschädigung des Biopsiekanales wahrscheinlich.

Das Verfahren verbindet die Möglichkeiten einer sowohl gezielten als auch ungezielten Probeentnahme, zum einen aufgrund einer lokalisierten Verletzung der Bronchialwand unter Röntgendurchleuchtung mit nachfolgender Zellaspiration, zum anderen durch Zellmobilisation von der Schleimhautoberfläche durch rasches Hin- und Herbewegen des Katheters.

Außer für die transbronchiale Nadelaspiration wurde bisher nur für die transbronchiale Katheteraspiration eine signifikant höhere Trefferquote beim peripheren Bronchialkarzinom für eine zytologische Methode im direkten Vergleich zur transbronchialen Zangenbiopsie beschrieben $[5,8,16]$.

\section{Transbronchiale Nadelaspiration}

Bei der transbronchialen Nadelaspiration handelt es sich um eine technisch fortgeschrittene und wenig invasive Methode, deren Komplikationsrate niedrig ist. Die Sensitivität der Methode ist hoch und in Studien gut belegt, allerdings wird sie wenig genutzt $[8,16,17]$. Gründe dafür sind das Fehlen technischer Fertigkeiten und die Sorge, den Arbeitskanal des Bronchoskopes zu zerstören $[18,19]$. Zudem verursacht das Verfahren im Vergleich zu Katheteraspiration und Bürstenabstrich höhere Kosten.

Für glatt begrenzte Läsionen ist die Trefferquote von transbronchialer Zangenbiopsie und transbronchialem Bürstenabstrich niedriger als die der transbronchialen Nadelaspiration [14]. In Fällen, in denen durch extrinsische Kompression der Bronchus deplatziert oder obstruiert ist, kann mit der Nadel die Bronchialwand durchstochen und der Tumor erreicht werden.

\section{Bronchiallavage}

Die höchste Trefferquote besteht bei exophytischem und infiltrativem Tumorwachstum im Vergleich zu submukösem oder extrabronchialem Wachstum. Bei der zytologischen Untersuchung von Sekreten besteht methodisch bedingt ein erhebliches quantitatives Missverhältnis zwischen der limitierten Menge der gewonnenen Proben und der Größe des Einzugsbereiches. Die diagnostische Sensitivität liegt in Untersuchungen mit peripheren Läsionen und Dominanz peripherer Bronchialkarzinome zwischen $4 \%$ und $40 \%[1,11,13,14,16,17]$.

Aus einer gezielten Probeentnahme mittels transbronchialer Nadelaspiration und Katheteraspiration resultiert eine deutlich höhere unabhängige diagnostische Sensitivität $[1,5,17]$. Die Methode der Bronchialwaschung sollte daher ausschließlich in der Kombination mit anderen Verfahren angewandt werden.

\section{Imprintzytologie}

Diese nimmt eine Sonderstellung ein, da histologische Präparate zusätzlich zytologisch untersucht werden. Dabei wird die Gewebeprobe leicht auf einen Objektträger getupft oder vorsichtig darüber gerollt, um Alterationen in der Zellmorphologie zu minimieren. Da durch das imprintzytologische und das histologische Präparat unterschiedliche Teile des Tumors erfasst werden, kann die Sensitivität in bezug auf Malignität gesteigert werden $[3,10,20]$.

\section{Schlussfolgerung}

Die Befragung zeigt, dass in einem hohen Prozentsatz (79\%) in pneumologischen Schwerpunktkliniken in Deutschland in der bronchoskopischen Diagnostik des peripheren Bronchialkarzi- 
noms die histologische Probeentnahme mit zusätzlicher Zytologie erfolgt, wodurch die Sensitivität bei gleichzeitig hoher Spezifität gesteigert werden kann. Die Methoden der Aspiration zytologischen Materials mittels Katheter oder Feinnadel werden jeweils nur in weniger als einem Drittel der Kliniken als häufigstes Verfahren praktiziert. Hinderungsgründe, die bei der Nadelaspiration ins Gewicht fallen, d. h. mangelnde Fertigkeit in der Handhabung, eine mögliche Beschädigung des Bronchoskopes sowie Materialkosten, treffen für die Katheteraspiration nicht zu. Da es sich um die einzigen Verfahren handelt, die eine signifikant höhere Trefferquote im Vergleich zur Zangenbiopsie aufweisen, sollten sie vermehrt zum Einsatz kommen.

\section{Literatur}

1 Baaklini WA, Reinoso MA, Gorin AB et al. Diagnostic yield of fiberoptic bronchoscopy in evaluating solitary pulmonary nodules. Chest 2000; 117: $1049-1054$

2 Popp W, Rauscher H, Ritschka L et al. Diagnostic sensitivity of different techniques in the diagnosis of lung tumors with the flexible fiberoptique bronchoscope. Cancer 1991; 67: 72 - 75

3 Welker L, Galle J, Vollmer E. Bronchologische Biopsiediagnostik des Bronchialkarzinoms - Zytologie und/oder Histologie? Pneumologie 2004; 58: $718-723$

4 Friedel H. Die Katheterbiopsie des peripheren Lungenherdes. Tuberkulosebibliothek Nr. 99. Leipzig: Barth, 1961

5 Franke $K-J$, Nilius G, Rühle $K-H$. Transbronchiale Biopsie im Vergleich zur Katheteraspiration bei peripheren Lungenherden. Pneumologie 2006; 60: 7-10

6 Agusti C, Xaubet A, Monton C et al. Induced sputum in the diagnosis of peripheral lung cancer not visible endoscopically. Respir Med 2001; 10: $822-828$

7 Chandan VS, Zimmerman K, Baker P et al. Usefulness of core roll preparations in immediate assessment of neoplastic lung lesions. Chest 2004; 126: 739 - 743

8 Gasparini S, Ferretti M, Secchi EB et al. Integration of transbronchial and percutaneous approach in the diagnosis of peripheral pulmonary nodules or masses. Chest 1995; 108: $131-137$
9 Lee $\mathrm{C}-\mathrm{H}$, Wang $\mathrm{C}-\mathrm{H}$, Lin $\mathrm{M}-\mathrm{C}$ et al. Multiple brushings with immediate Rius stain via flexible fibreoptic bronchoscopy without fluoroscopic guidance in the diagnosis of peripheral pulmonary tumours. Thorax 1995; 50: $18-21$

10 Liao W-Y, Jerng J-S, Chen $K-Y$ et al. Value of imprint cytology for ultrasound-guided transthoracic core biopsy. Eur Respir J 2004; 24: 905 909

11 Mak VHF, Johnston IDA, Hetzel MR et al. Value of washings and brushings at fibreoptic bronchoscopy in the diagnosis of lung cancer. Thorax 1990; 45: 373-376

12 Rosell A, Monso E, Lores $L$ et al. Cytology of bronchial biopsy rinse fluid to improve the diagnostic yield for lung cancer. Eur Respir J 1998; 12 : $1415-1418$

13 Bilaceroglu S, Kumcuoglu Z, Alper $H$ et al. CT bronchus sign-guided bronchoscopic multiple diagnostic procedures in carcinomatous solitary pulmonary nodules and masses. Respiration 1998; 65: 49-55

14 Chechani V. Bronchoscopic diagnosis of solitary pulmonary nodules and lung masses in the absence of endobronchial abnormality. Chest 1996; 109: 620-625

15 Drift MA van der, Wilt G-J van der, Thunnissen FBJM et al. A prospective study of the timing and cost-effectiveness of bronchial washing during bronchoscopy for pulmonary malignant tumors. Chest 2005; 128: $394-400$

16 Katis $K$, Inglesos E, Zachariadis E et al. The role of transbronchial needle aspiration in the diagnosis of peripheral lung masses or nodules. Eur Respir J 1995; 8: 963 - 966

17 Reichenberger F, Weber J, Tamm $M$ et al. The value of transbronchial needle aspiration in the diagnosis of peripheral pulmonary lesions. Chest 1999; 116: 704-708

18 Haponik EF, Shure D. Underutilization of transbronchial needle aspiration: experiences of current pulmonary fellows. Chest 1997; 112: $251-253$

19 Prakash UB, Offord KF, Stubbs SE. Bronchoscopy in North America: the ACCP survey. Chest 1991; 100: 1668 - 1675

20 Kawaraya M, Gemba K, Ueoka H et al. Evaluation of various cytological examinations by bronchoscopy in the diagnosis of peripheral lung cancer. Br J Cancer 2003; 89: 1885 - 1888 